\section{Our experts highlight the most important research articles across the spectrum of topics relevant to the field of hepatic oncology}

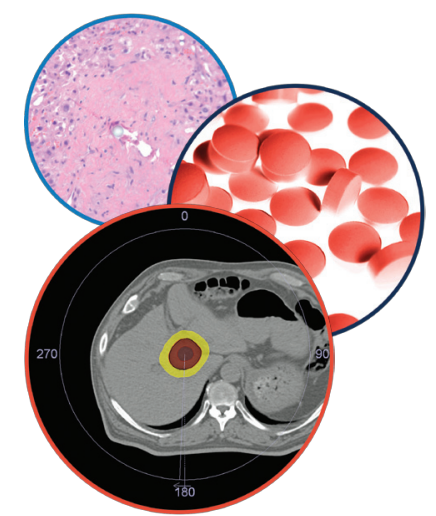

Hepatic Oncology

Expert panel: Alexsander K Bressan, Department of Surgery, University of Calgary \& the Foothills Medical Centre, Alberta, Canada; Chad G Ball, Department of Surgery, University of Calgary \& the Foothills Medical Centre, Alberta, Canada; Elijah Dixon, Department of Surgery, University of Calgary \& the Foothills Medical Centre, Alberta, Canada

Moulton CA, Gu CS, Law CH et al.

Effect of PET before liver resection on surgical management for colorectal adenocarcinoma metastases:

a randomized clinical trial. JAMA 311(18), 1863-1869 (2014).

Many studies have demonstrated superior accuracy of PET-CT, compared with MRI and CT, in identifying hepatic metastasis from colorectal cancer. The added value in surgical management, however, is still controversial. Reported ability to change surgical planning ranges from 20 to $30 \%$, and derives mostly from small retrospective studies.

Moulton and colleagues conducted a randomized controlled trial including 404 patients with resectable colorectal liver metastasis and resected primary tumor from nine centers in Canada, over an 8-year period (2005-2013). Participants were randomized to include or not PET-CT on standard workup prior to liver resection, with the primary objective of assessing change in surgical planning based on PET-CT findings.

New information was provided in 111 patients (42.2\%), with additional lesions in 49 cases. Change in surgical planning was indicated in only 21 patients $(8.0 \%)$. Secondary outcome analysis did not show statistically significant difference in overall or disease-free survival, with a median follow-up of 36 months .

This is the largest randomized trial on this subject to date, and it supports a more conservative use of PET-CT. Reproduction of its results is necessary, with emphasis on preoperative use of chemotherapy and time interval to PET-CT.
Cost-effectiveness analysis of selective indication of PET-CT for patients with high risk for futile laparotomies is also needed.

Primrose J, Falk S, Finch-Jones M et al. Systemic chemotherapy with or without cetuximab in patients with resectable colorectal liver metastasis: the new EPOC randomized controlled trial. Lancet Oncol. 15(6), 601-611 (2014).

Primrose and colleagues reported interim results of a multicentric trial in the UK, evaluating the effect of adding cetuximab to perioperative chemotherapy in patients with colorectal liver metastases. Patients with resectable or suboptimally resectable liver metastases were enrolled from February 2007 to November 2012; a protocol amendment restricted eligibility to patients with $K R A S$ exon 2 wildtype cancers after July 2008. A total of 272 patients were randomized to perioperative chemotherapy with or without cetuximab.

Completion of all planned cycles in the chemotherapy plus cetuximab group and chemotherapy-alone group was, respectively, 76 and $73 \%$ for preoperative, and 50 and $47 \%$ for postoperative chemotherapy. Resection was possible in $87 \%$ of patients receiving cetuximab and $93 \%$ in the control group. With overall median follow-up of 20.7 months , median progressionfree survival was inferior in the cetuximab group (14.1 vs 20.5 months; HR: 1.48, 95\% CI: 1.04-2.12), defining closure of the study protocol in November 2012.

Skepticism has replaced much of the enthusiasm around the use of biologic agents
Future Medicine part of 
in the adjuvant treatment of colorectal liver metastasis. Increased costs and toxicity have been weakly supported by a modest survival benefit demonstrated in the palliative setting. The unexpected results of this study raise additional safety concerns about off-label use of new drugs. Enrollment in clinical trials and oversight by data monitoring committees must be encouraged.

Hyder O, Marques H, Pulitano C et al. A nomogram to predict long-term survival after resection for intrahepatic cholangiocarcinoma: an Eastern and Western experience. JAMA Surg. 149(5), 432-438 (2014).

The 7th edition of TNM introduced a new staging classification for intrahepatic cholangiocarcinoma, distinct from hepatocellular carcinoma. This separation was centered in the exclusion of tumor size for T2 vs T3 classification, as supported by population-based registry data. Additional insight from surgical series is necessary to validate and refine this new staging version.

Hyder and colleagues conducted an international multi-institutional study including 514 patients with resected intrahepatic cholangiocarcinomas at 13 centers in the US, Europe and Asia from 1990 to 2011. Clinical and pathologic prognostic factors were identified and combined into a nomogram to predict overall survival.

Tumor size, age, multiple lesions, nodal status, vascular invasion and presence of cirrhosis were independently associated with survival on regression analysis. Non-linear effect of tumor size and age on survival was taken into account. Predictive accuracy of the model was superior to the 7 th edition of TNM ( $C$ statistic of 0.69 and 0.59 , respectively).

This study stands out by its multiinstitutional heterogeneous population, the careful modeling of continuous variables and improved accuracy of the resultant prediction nomogram. These distinctive characteristics may still justify the revisited association of tumor size with prognosis. While external validation is awaited, interest in additional studies on the prognostic relevance of tumor size seems already warranted.

Financial \& competing interests disclosure

The authors have no relevant affiliations or financial involvement with any organization or entity with a financial interest in or financial conflict with the subject matter or materials discussed in the manuscript. This includes employment, consultancies, honoraria, stock ownership or options, expert testimony, grants or patents received or pending, or royalties.

No writing assistance was utilized in the production of this manuscript. 